\title{
Projet d'entreprise et prospective : l'expérience d'un organisme public de recherche, le CIRAD
}

\section{Marie de Lattre-Gasquet}

\section{Résumé}

Le CIRAD (Centre de coopération internationale en recherche agronomique pour le développement), établissement public français de recherche agricole travaillant en coopération avec les pays du Sud, intervient dans plus de cinquante pays, il doit constamment s'adapter aux évolutions en cours afin de remplir au mieux sa mission. Au cours des cinq dernières années, à deux reprises, le CIRAD s'est donné les moyens de préparer l'avenir. II a d'abord élaboré un projet d'entreprise (1989-1991), puis a réfléchi à plusieurs options stratégiques en fonction de différentes évolutions de l'environnement (1993-1994). Dans les deux cas, le CIRAD a fait appel à des consultants qui ont aidé à éviter de ressasser les idées et les convictions qui imprègnent l'organisme et ont permis aux groupes de conception interne d'être plus imaginatifs.

En comparant les deux exercices (projet d'entreprise et réflexion prospective et stratégique), on observe que plus la démarche d'élaboration est participative, plus l'appropriation et l'action se font bien, qu'il s'agisse du personnel du CIRAD, des responsables dans les ministères ou des partenaires de recherche dans les pays en développement et dans les pays développés. Néanmoins, le fait pour le CIRAD d'être un centre de recherche a pu contribuer à rendre difficile le passage de la réflexion prospective à des choix stratégiques.

\section{Citer ce document / Cite this document :}

de Lattre-Gasquet Marie. Projet d'entreprise et prospective : l'expérience d'un organisme public de recherche, le CIRAD. In: Politiques et management public, vol. 15, $n^{\circ} 4$, 1997. pp. 47-63;

doi : https://doi.org/10.3406/pomap.1997.2164

https://www.persee.fr/doc/pomap_0758-1726_1997_num_15_4_2164

Fichier pdf généré le 22/04/2018 


\title{
PROJET D'ENTREPRISE ET PROSPECTIVE : L'EXPERIENCE D'UN ORGANISME PUBLIC DE RECHERCHE, LE CIRAD
}

\author{
Marie de LATTRE-GASQUET ${ }^{*}$
}

Résumé

Le CIRAD (Centre de coopération internationale en recherche agronomique pour le développement), établissement public français de recherche agricole travaillant en coopération avec les pays du Sud, intervient dans plus de cinquante pays. II doit constamment s'adapter aux évolutions en cours afin de remplir au mieux sa mission. Au cours des cinq dernières années, à deux reprises, le CIRAD s'est donné les moyens de préparer l'avenir. Il a d'abord élaboré un projet d'entreprise (1989-1991), puis a réfléchi à plusieurs options stratégiques en fonction de différentes évolutions de l'environnement (1993-1994). Dans les deux cas, le CIRAD a fait appel à des consultants qui ont aidé à éviter de ressasser les idées et les convictions qui imprègnent l'organisme et ont permis aux groupes de conception interne d'être plus imaginatifs.

En comparant les deux exercices (projet d'entreprise et réflexion prospective et stratégique), on observe que plus la démarche d'élaboration est participative, plus l'appropriation et l'action se font bien, qu'il s'agisse du personnel du CIRAD, des responsables dans les ministères ou des partenaires de recherche dans les pays en développement et dans les pays développés. Néanmoins, le fait pour le CIRAD d'être un centre de recherche a pu contribuer à rendre difficile le passage de la réflexion prospective à des choix stratégiques.

- CIRAD.

Revue POLITRUES ET MANAGEMENT PUBLIC, Volume 15, n 4, décembre 1997.

(C) Institut de management Public - 1997. 
Les responsables d'entreprise sont constamment a la recherche de nouvelles méthodes pour mieux mobiliser les ressources vers les objectifs. Dans les années 80 , ils ont fait faire des projets d'entreprise pour créer ou renforcer une culture commune et ont établi des plans stratégiques pour guider leur action. Depuis le début des années 90 , des sociétés industrielles et de service ont une activité de prospective (Lesourne et Stoffaěs, 1996 ; Benassouli et Monti, 1995). Elles comprennent combien il est nécessaire de discerner comment pourrait évoluer à moyen et à long terme leur environnement, mais également combien il est dangereux d'accorder une confiance excessive à des prévisions chiffrées élaborées en faisant l'impasse sur un grand nombre de variables qualitatives que l'on ne sait pas intégrer dans les modèles. Le Centre de coopération internationale en recherche agronomique pour le développement (CIRAD) a jugé utile, pour conduire son action, d'élaborer un projet d'entreprise et de faire ensuite un exercice de prospective. Le présent article s'attache à présenter et discuter ces deux démarches mises en œuvre par une entreprise publique de recherche agricole intervenant dans plus de cinquante pays.

Le CIRAD, un établissement public de recherche agricole travaillant en cooperation avec les pays du Sud
Etablissement public à caractère industriel et commercial (EPIC), le Centre de coopération internationale en recherche agronomique pour le développement (CIRAD) a été créé en 1985 à partir d'instituts eux-mêmes constitués pendant la période coloniale. Ces instituts avaient pour mission d'entreprendre des recherches afin d'améliorer les productions agricoles, forestières et animales dans les régions tropicales et de développer leurs débouchés sur les marchés locaux ou à l'exportation.

Au moment de sa création, le CIRAD comptait onze départements, jusqu'alors instituts indépendants, ayant des statuts différents : association, société d'Etat ou établissement public. Le CIRAD est placé sous la co-tutelle des ministères chargés de la recherche et de la coopération. II emploie 1800 personnes (dont 900 chercheurs). Plus du tiers du personnel est en poste outre-mer, réparti dans une cinquantaine de pays, et travaille dans des institutions publiques de recherche, des sociétés de développement et des entreprises agro-alimentaires et agro-industrielles. Environ les deux tiers de son budget proviennent du budget civil de recherche et de développement technologique (BCRD) ; le reste provient de la vente de produits, de marchandises et de prestations de service.

En novembre 1995, dans son rapport d'instruction, le Comité national d'évaluation de la recherche (CNER) note que la première impression qui se dégage de l'analyse très factuelle des missions, de l'organisation, des modes de fonctionnement et de l'activité du CIRAD est celle d'une grande perplexité face à un organisme complexe, pétri de contradictions originelles que l'absence d'une stratégie gouvernementale nettement affichée n'a guère clarifiée, marqué par une histoire qui continue à tracer son avenir. II existe en particulier une interrogation sur la possibilité de concilier les trois termes du mandat : recherche, développement et coopération. La deuxième impression est celle d'un organisme vivant, dynamique et original, dont la première des caractéristiques est 
de vouloir et de pouvoir répondre aux demandes, souvent mal formulées, de ses partenaires après les avoir traduites en opérations de recherche et de développement et s'être assuré les financements nécessaires à leur mise en œuvre.

Le CIRAD se trouve dans un environnement où les facteurs économiques et politiques (français et extérieurs) changent rapidement. Pour mobiliser l'ensemble du personnel sur des objectifs clairs et partagés, il doit les analyser et, dans la mesure du possible, prévoir leur évolution afin d'être pro-actif, c'est-à-dire agir pour anticiper les changements plutôt que les subir. C'est la raison pour laquelle, en 1989, le CIRAD a commencé à préparer un projet d'entreprise; puis, en 1993, s'est lancé dans un exercice de prospective.

Pour un nouveau CIRAD : le projet d'entreprise
En 1989, le ministère chargé de la Recherche et de la Technologie a demandé à différents organismes de recherche de préparer un plan d'établissement. Ce plan ne devait être "ni un contrat-plan, ni un schéma glissant, mais plutôt une réflexion collective sur les missions de l'établissement, l'analyse de ses forces et de ses faiblesses, et la détermination d'un projet collectif visant à mettre en place une politique à court, moyen et long-terme". Constatant que l'institution créée quatre ans plus tôt était en fait la somme de départements avec chacun sa culture, son identité, ses objectifs de recherche, ses partenaires scientifiques et de développement, et ses sources de financement, le directeur général du CIRAD a décidé de transformer la demande du ministre de la Recherche. II est passé du "plan d'établissement", qui semblait rigide et lié à une culture de la fonction publique, au "projet d'entreprise". Une démarche participative a été adoptée et la philosophie de l'établissement et ses grands objectifs ont été formulés pour qu'ils deviennent une référence pour l'action (Gondrand, 1987). La spécificité du statut d'établissement à caractère industriel et commercial, et donc la logique d'entreprise, ont été affichées.

En faisant un projet d'entreprise, la direction générale avait quatre objectifs. Premièrement, il s'agissait de répondre à la demande du ministre. Deuxièmement, il fallait établir le CIRAD. Le CIRAD avait été créé quatre ans plus tôt à partir de la fusion d'instituts indépendants et, en 1989, les cultures des départements prévalaient sur celle de linstitution. Chaque département avait son nom et son logo, et de nombreux membres du personnel ignoraient même la signification du sigle CIRAD. II était nécessaire d'intégrer et de transformer ces cultures afin d'établir une culture CIRAD. Troisièmement, il était nécessaire de s'adapter à un nouveau contexte de coopération scientifique Nord-Sud. Depuis la création du CIRAD, les institutions de recherche des pays en développement s'étaient renforcées, les centres internationaux de recherche travaillaient avec ces institutions nationales et, en Europe, la recherche agricole pour le développement commençait à se structurer. Le quatrième objectif était externe. II s'agissait d'améliorer la lisibilité et l'image de l'institution en offrant aux partenaires une stratégie transparente, d'amener le CIRAD à un niveau international en publiant un document de qualité équivalente à celle des plans à moyen terme des centres 
intemationaux du Groupe consultatif pour la recherche agricole internationale (GCRAI) et, par conséquent, de montrer que la création du CIRAD avait apporté une valeur ajoutée par rapport à la situation antérieure et permettait de mieux répondre aux enjeux du développement.

La préparation du projet d'entreprise a été faite à partir de deux principes : le projet doit naître des aspirations et de l'expérience du personnel ; le projet doit préparer le CIRAD à l'avenir. Le processus d'élaboration a été par conséquent très participatif, ce qui a joué un grand rôle dans son acceptation. En effet, comme le dit Hernandez (1994), le contenu proprement dit du projet d'entreprise importe moins que les conditions de sa naissance et de son utilisation ultérieure dans l'entreprise.

La première phase de la préparation du projet d'entreprise a consisté en une analyse de l'environnement du CIRAD portant sur les principaux risques de déséquilibre dans l'évolution à long et moyen terme des agricultures, des ressources naturelles et des secteurs agro-industriels et agro-alimentaires du monde. Elle a comporté également une analyse de la situation interne de l'établissement. Un groupe d'une vingtaine de cadres dirigeants et de chercheurs, aidé d'un consultant (Jacques Giri), y a réfléchi. La méthode de travail a été relativement informelle, comparée à la méthode de prospective par scénarios qui sera utilisée par la suite. Des documents de travail ont été écrits par les membres du groupe, puis synthétisés par le consultant. Les instances de représentation interne, les tutelles et les principaux partenaires ont été consultés.

Après une année de travail, un document intitulé Un projet d'entreprise pour ke CIRAD. Quel avenir ? Quelle stratégie ? a été publié sous une forme provisoire et envoyé à tous les membres du personnel. De façon concomitante, une cassette vidéo a été réalisée pour présenter les idées principales du document et permettre au personnel, en particulier aux expatriés, de mieux participer au débat. Avec ces deux outils (document et cassette vidéo), la seconde phase de préparation du projet d'entreprise a pu démarrer. II s'agissait de consulter tout le personnel du CIRAD et ses partenaires, de faire la synthèse de leurs remarques et de les intégrer dans un document final, puis de faire approuver le projet et la stratégie qui en découlait par les instances statutaires. Dans les mois qui ont suivi, quatre cents personnes, soit presque un quart du personnel, ont donné leur point de vue sur l'analyse de l'environnement, soit de façon individuelle, soit de façon collective. Le document et la cassette vidéo ont été également présentés à de nombreux partenaires, qui ont pu donner leur avis. Trois éléments ont permis ce fort taux de réponse : le texte était facile à lire, le délai de réaction était long, et toute l'équipe de direction générale était engagée dans le processus. Toutes les suggestions ont été analysées et synthétisées et, dans la mesure du possible, prises en compte. Moins de six mois plus tard, un document final, intitulé Le Projet d'entreprise du CIRAD. Renouveler notre coopération dans un monde qui change, était publié.

Le premier document synthétisait une analyse de l'environnement externe, des demandes potentielles futures, de l'offre du CIRAD, des relations et des alliances à 
nover, et de la situation interne. La stratégie future était à peine esquissée. Dans le second document, par contre, des directions stratégiques pour les années 90 étaient indiquées. Elles ont pu étre définies car un accord général avait été établi sur le bilan de la situation interne et externe.

Pour favoriser l'adhésion formelle des tutelles au projet d'entreprise et son appropriation par l'ensemble du personnel afin qu'il s'applique à mettre en œuvre les recommandations stratégiques, en septembre 1991 - soit deux ans après le début de l'exercice -, sous la présidence du ministre de la recherche, deux journées ont été consacrées au projet et à son exécution. Six ateliers ont été constitués, dont les membres ont réfléchi aux modalités de mise en œuure des idées émises. Dans les mois qui ont suivi, la direction générale a décidé d'importantes réformes : création de nouveaux départements établis à partir de la fusion de départements héritiers des anciens instituts, suppression des logos des anciens départements et adoption d'un logo unique pour l'institution, lancement de deux journaux d'entreprise et renouvellement des publications, nouvelles alliances internationales, etc. Ces réformes structurelles, a priori peu populaires, ont été acceptées grâce à l'approche participative choisie et à un effort de communication. Elles ont permis à la culture CIRAD d'émerger. Les objectifs internes ont par conséquent été atteints.

Le projet d'entreprise a été également diffusé à l'extérieur du CIRAD. L'objectif était de montrer qu'il y avait un nouveau CIRAD qui n'était pas la somme des instituts créés pendant la période coloniale, mais qu'il avait sa propre identité et était à l'écoute des souhaits et demandes des partenaires des pays en développement, de la France et de l'Europe. Il s'agissait de donner une image professionnelle et intemationale au CIRAD. II fallait montrer qu'une autre recherche agricole française pour les tropiques était née. La démarche de consultations externes et la large diffusion du document ont produit cet effet.

Dans les ministères de tutelle, le document a été reconnu comme étant de qualité, mais peu de mesures ont été prises pour aider le CIRAD à mettre en œuvre certaines de ses orientations stratégiques.

Le projet du CIRAD n'a pas été imposé. II a été l'expression d'un premier groupe, mais il a été travaillé, discuté, reformulé par l'ensemble du corps social, qui a ainsi participé au développement de la culture de l'entreprise et à la socialisation de son identité (Reitter et Ramanantsoa, 1985). II y a eu un processus itératif entre les différentes composantes du corps de l'entreprise qui ont tenté de répondre à des questions aussi fondamentales que : qui sommes-nous, quels sont nos objectifs, nos atouts, nos faiblesses, quelles valeurs devons-nous développer dans ce sens ?, etc. Thiétart (1984) appelle cela un processus de planification stratégique "haut-bas-haut". La philosophie du CIRAD pour l'avenir a été résumée en une phrase : renouveler notre coopération dans un monde qui change. Les objectifs du projet d'entreprise, qui étaient de créer une identité CIRAD et une culture d'entreprise, ont été atteints sur le plan interne et sur le plan international. 
Mais, la démarche a eu des limites. Le projet d'entreprise du CIRAD était un produit un peu hybride, à la fois projet d'entreprise et plan stratégique. Comme un projet d'entreprise classique, il a cherché à créer un sentiment d'appartenance (en rappelant I'histoire vécue ensemble), à développer un consensus minimal (en déclinant les valeurs qui guident l'action), à donner un sens à l'activité de chacun (en fixant des règles du jeu dans la gestion du quotidien). Mais, comme un plan stratégique, il a indiqué les grandes orientations du CIRAD pour l'avenir ainsi que l'intensité de l'engagement. La deuxième limite réside dans son contenu. Certaines ambiguĩtés n'ont pas été levées. Ainsi, la régression de l'expatriation n'a pas été admise; le positionnement entre l'Europe et le multilatéral est resté flou; les relations avec les entreprises et les activités commerciales ont été à peine esquissées. La troisième limite est institutionnelle. Pour exploiter la dynamique du changement ainsi créée, le CIRAD a manqué de relais chez ses tutelles.

Think global and act local : un exercice de prospective par scénarios
En mars 1993, constatant de nombreux changements dans l'environnement et dans les positions des acteurs externes, ainsi que les difficultés à atteindre certains des objectifs définis dans le projet d'entreprise, le directeur général du CIRAD a demandé à un groupe interne de mener un travail sur les évolutions de l'environnement de l'organisme, les forces et les faiblesses du CIRAD, et de proposer des options stratégiques. C'est ainsi que le groupe prospective et stratégie a été établi. II a regroupé une vingtaine de chercheurs originaires de tous les départements du CIRAD sur une durée d'un an et demi (de mars 1993 à novembre 1994). Le groupe prospective et stratégie a bénéficié dans sa démarche de l'appui de deux consultants du GERPA (Groupe d'études ressources, prospective, aménagement). Il a en partie suivi la méthode des scénarios décrite par Michel Godet (1991) et utilisé certains de ses outils.

La prospective, c'est l'anticipation pour guider l'action. Cette indiscipline intellectuelle (Pierre Massé, 1965) s'attache à voir loin, large et profond (Gaston Berger) mais aussi autrement et ensemble. En d'autres termes, la vision globale, volontariste et à long terme s'impose pour donner un sens à l'action. En établissant un groupe prospective et stratégie, la direction générale avait deux objectifs. Le premier objectif était opérationnel : il s'agissait de rassembler des propositions de recommandations stratégiques pour un positionnement de l'entreprise dans son environnement et de contribuer ainsi à construire le plan stratégique du CIRAD. Le second objectif était institutionnel : il s'agissait de développer au sein du centre un réflexe prospectif face à un contexte en mutation rapide.

Le groupe prospective et stratégie a mené son travail en cinq étapes, qui ont conduit à la publication d'un rapport interne (1995), diffusé au personnel et aux partenaires du CIRAD afin de recueillir leurs réactions. Le tableau 1 résume la démarche. 


\section{Tableau 1 : La démarche du groupe prospective et stratégle}

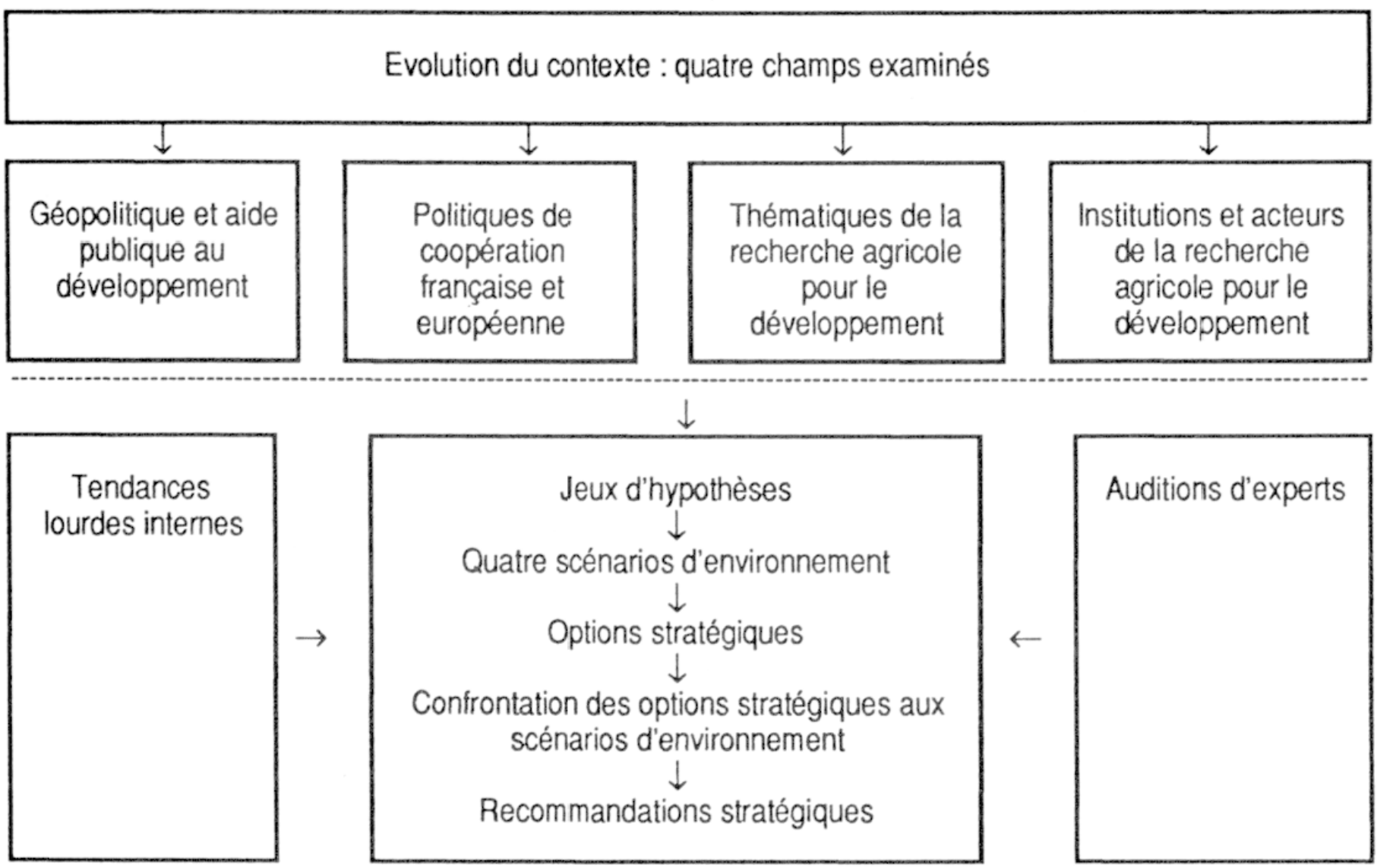

La première étape du travail a consisté à essayer de se poser les bonnes questions et à identifier les variables de toute nature, qui exercent ou sont susceptibles d'exercer une influence sur le problème étudié (de Jouvenel, 1993). Pour ce faire, les membres du groupe et les consultants se sont réunis dans un atelier de prospective pendant deux jours pour amorcer la dynamique du groupe, découvrir les problèmes et commencer la réflexion. II s'agissait, d'une part, de réunir, puis de hiérarchiser un maximum d'idées sur l'ensemble des facteurs de changement et sur les inerties qui affecteront l'avenir du CIRAD à l'horizon de dix ans, d'autre part, de recenser l'ensemble des idées reçues qui circulent dans le domaine de la recherche agricole pour le développement. Les participants à l'atelier de prospective ont eu une grande liberté de parole, ce qui a permis de lancer le groupe et de motiver ses membres. Quatre domaines ont été identifiés comme clefs pour l'avenir du CIRAD : les espaces géopolitiques du Sud, qui sont ses terrains d'intervention; les institutions et les acteurs de la recherche agricole pour le développement, qui sont, ou seront demain, ses partenaires mais aussi ses concurrents ; les thématiques scientifiques, ses domaines de compétence ; les produits et marchés agricoles et agro-industriels, ses objets de recherche. 
Encouragé par les consultants, le groupe prospective et stratégie a tenté de faire une analyse structurelle, qui permet de décrire un système à l'aide d'une matrice mettant en relation tous ses éléments constitutifs et ainsi de faire apparaitre les variables essentielles de l'évolution du système. Mais la longueur du processus ( 6 à 8 mois), la complexité des missions du CIRAD, des modalités d'intervention et des contextes ont rendu ce travail difficile et fastidieux. II a donc été décidé d'abandonner cette méthode et de constituer une base d'analyse, puis de faire des choix. Une cinquantaine de fiches ont été réalisées. Elles proposaient une analyse rétrospective et actuelle de chaque variable, indiquaient les facteurs déterminants, les tendances, les éléments possibles de rupture et les facteurs de changement. Leur rédaction a été l'occasion d'expliciter certaines questions, ou d'améliorer la connaissance de marchés, de régions géographiques et d'acteurs de la recherche et du développement. La construction de cette base d'analyse a permis de déterminer les variables-clefs d'évolution de l'environnement du CIRAD, et d'analyser le passé et la stratégie des acteurs.

La définition des domaines importants pour l'environnement du CIRAD a été affinée : la géopolitique et l'aide publique au développement, les politiques de coopération française et européenne, les thématiques de la recherche agricole pour le développement, les institutions et acteurs. Pour chacun des quatre domaines, les tendances lourdes, les incertitudes majeures et les ruptures possibles ont été identifiées. Des jeux d'hypothèses (un jeu d'hypothèse tendanciel et plusieurs jeux d'hypothèses contrastées) ont été proposés. Le tableau 2 résume le travail sur les thématiques de la recherche agricole pour le développement.

La deuxième étape a consisté à analyser le jeu des acteurs de l'univers de la recherche-développement. La méthode MACTOR (Méthode, acteurs, objectifs, rapports de force), conçue et développée par Michel Godet, a été utilisée au cours de deux ateliers. La méthode comporte six phases:

1. construction du tableau de stratégie des acteurs moteurs, c'est-à-dire ceux qui ont une influence sur les variables clefs préalablement définies, afin de représenter les finalités de chaque acteur, ses contraintes et ses moyens d'action ;

2. identification des enjeux stratégiques et des objectifs associés pour chaque acteur moteur ;

3. évaluation de la position de chaque acteur par rapport aux pistes stratégiques et aux objectifs opérationnels; des matrices acteurs $x$ objectifs sont construites ; 
Tableau 2 : Thématiques de la recherche agricole pour le développement

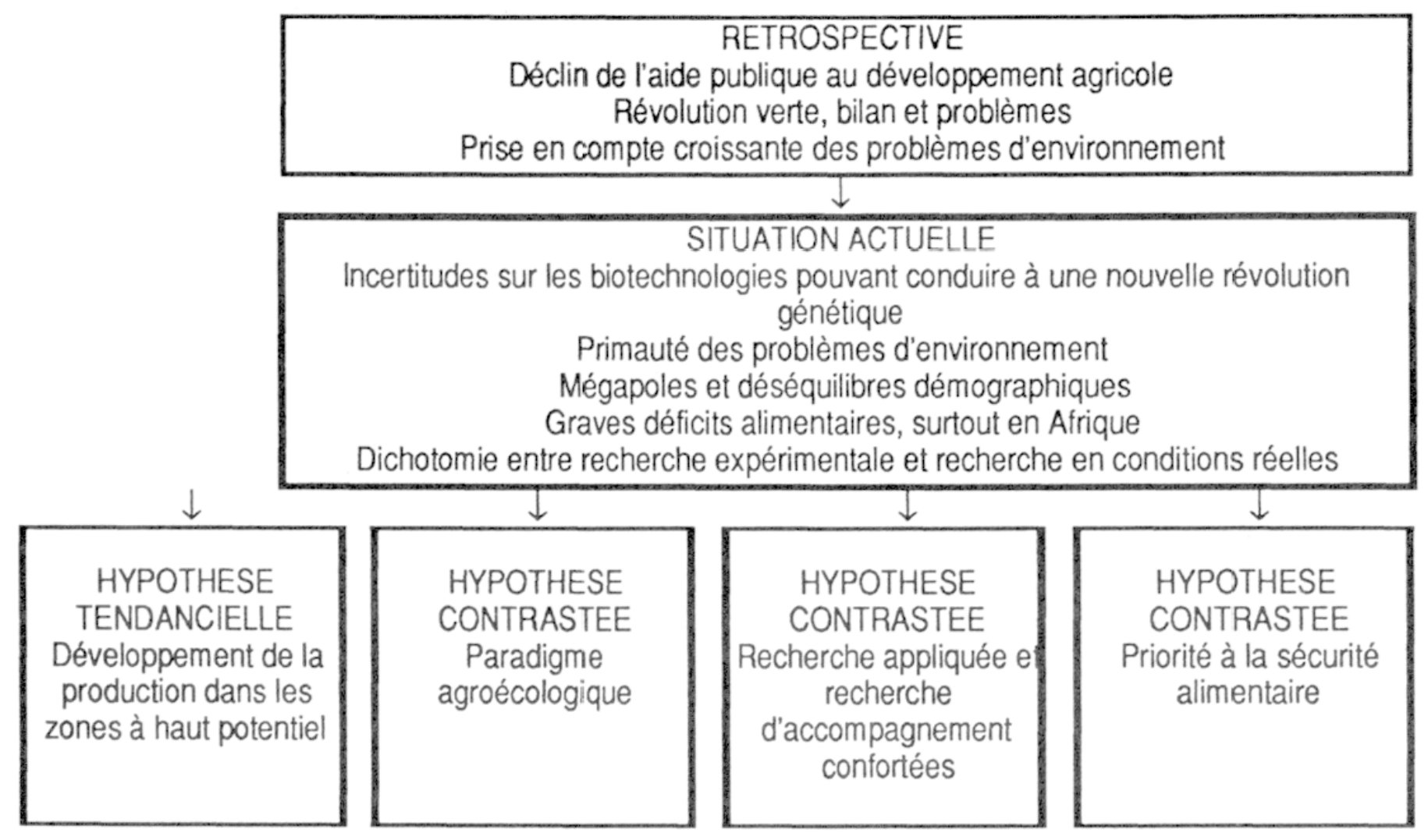

4. recensement des alliances entre les acteurs et des conflits potentiels en fonction des objectifs stratégiques ; les graphes des convergences et des divergences possibles entre acteurs sont obtenus, ce qui permet de visualiser des groupes d'acteurs (convergence d'intérêt) et leur degré de liberté apparent, de repérer les acteurs les plus menacés potentiellement, et d'analyser la stabilité du système ;

5. représentation des rapports de force entre acteurs ;

6. questions-clefs pour l'avenir.

A partir des jeux d'hypothèses sur les variables clefs externes et sur les jeux d'acteurs, quatre scénarios d'évolution de l'environnement ont été construits. Ces scénarios sont : les évolutions tendancielles se poursuivent (scénario $A$ ) ; une nouvelle division de la recherche agricole internationale se met en place (scénario B) ; la France et l'Union européenne s'engagent dans une politique africaine volontariste (scénario C) ; la recherche agricole publique se paupérise (scénario D).

En principe, la troisième étape doit permettre de balayer le champ des possibles et de réduire l'incertitude. Elle est généralement réalisée avec l'aide d'experts et appuyée par d'autres méthodes plus ou moins sophistiquées (méthode Delphi, méthode 
d'impacts croisés). Les membres du groupe prospective et stratégie ont décidé d'avoir des entretiens avec une trentaine de personnalités françaises et étrangères extérieures au CIRAD et de tester auprès d'elles les scénarios d'évolution de l'environnement de l'institution, qui ont été ensuite corrigés.

Au cours de la quatrième étape, les tendances lourdes internes de linstitution doivent être identifiées. Au CIRAD, le directeur général avait demandé à avoir un éclairage sur l'environnement de l'institution, et le mandat du groupe prospective et stratégie était donc limité à cet aspect. L'analyse de la situation interne était par ailleurs faite par d'autres, et elle n'a donc pas fait l'objet d'investigations particulières. Néanmoins, les membres du groupe ont pensé que, dans un souci de cohérence méthodologique, un chapitre devait être rédigé sur ce sujet, et il l'a été à partir d'éléments rassemblés par d'autres personnes.

Durant la cinquième étape, les options stratégiques sont évaluées et choisies. Le groupe avait étudié les évolutions de l'environnement du CIRAD, défini des hypothèses et proposé des scénarios pour l'avenir. Les tendances lourdes internes avaient été identifiées dans les domaines sensibles de la gestion d'entreprise. Le croisement des diagnostics d'environnement et de l'évaluation interne a conduit, d'une part, à identifier des enjeux et des défis à relever pour le CIRAD dans les dix prochaines années, d'autre part, à proposer trois options stratégiques contrastées décrivant des positionnements possibles pour le CIRAD en fonction de certaines hypothèses d'évolution de l'environnement. Les options stratégiques étaient : (1) une stratégie euro-africaine en coopération ; (2) une dynamique commerciale et une logique de marché ; (3) le pilotage par la logique scientifique et les grands programmes internationaux.

La confrontation des options stratégiques aux scénarios d'environnement a permis de mesurer leur flexibilité et leur robustesse. Une option est robuste si elle peut être jouée avec succès dans la majorité des scénarios d'environnement. Elle est risquée si elle est incompatible avec certains scénarios. Elle est flexible si elle peut être jouée avec des résultats acceptables quel que soit le scénario envisagé. Le tableau 3 résume la confrontation des options stratégiques aux scénarios d'environnement. Aucune option stratégique n'était compatible avec l'ensemble des quatre scénarios d'évolution de l'environnement. Cependant, chacune des options était bien adaptée à un scénario d'environnement donné : I'option 1 (stratégie euro-africaine) au scénario C (politique française et européenne en coopération); l'option 2 (dynamique commerciale) au scénario D (paupérisation de la recherche publique) ; I'option 3 (logique scientifique) au scénario B (nouvelle division de la recherche agronomique internationale). Le groupe prospective et stratégie a conclu ses travaux en formulant quelques recommandations stratégiques pour chacun des grands domaines d'activité qui composent le métier du CIRAD. Le groupe n'avait qu'un rôle d'anticipation ; l'acte stratégique est de la responsabilité de la direction. 
Projet d'entreprise et prospective : l'expérience d'un organisme

public de recherche, le CIRAD

Tableau 3 : Confrontation des options stratégiques aux scénarios d'environnement

\begin{tabular}{|c|c|c|c|c|c|}
\hline Stratégies & $\begin{array}{l}\text { Scénario A } \\
\text { Tendanciel }\end{array}$ & \begin{tabular}{|c} 
Scénario B \\
Nouvelle division \\
recherche \\
agricole \\
internationale
\end{tabular} & $\begin{array}{c}\text { Scénario C } \\
\text { Politique française } \\
\text { et européenne en } \\
\text { coopération }\end{array}$ & $\begin{array}{c}\text { Scénario D } \\
\text { Paupérisation } \\
\text { et dissolution } \\
\text { de la recherche } \\
\text { agricole } \\
\text { publique }\end{array}$ & Résultat \\
\hline $\begin{array}{l}\text { Option 1 } \\
\text { Stratégie euro- } \\
\text { africaine }\end{array}$ & $\begin{array}{c}+ \\
\text { (1) }\end{array}$ & (3) & $\begin{array}{l}++ \\
(1)\end{array}$ & $\begin{array}{c}+/++ \\
(1)\end{array}$ & $\begin{array}{c}\text { Stratégie } \\
\text { assez robuste }\end{array}$ \\
\hline $\begin{array}{c}\text { Option } 2 \\
\text { Rationalisation } \\
\text { et dynamique } \\
\text { commerciale }\end{array}$ & $\begin{array}{l}0 /+ \\
(2)\end{array}$ & $\begin{array}{l}0 /+ \\
(2)\end{array}$ & $\begin{array}{r}+ \\
\text { (2) }\end{array}$ & $\begin{array}{l}+/++ \\
\text { (1) }\end{array}$ & $\begin{array}{l}\text { Stratégie } \\
\text { flexible }\end{array}$ \\
\hline $\begin{array}{l}\text { Option } 3 \\
\text { Pilotage par } \\
\text { la logique } \\
\text { scientifique et } \\
\text { internatio- } \\
\text { nalisation }\end{array}$ & $\begin{array}{l}0 /+ \\
(2)\end{array}$ & $\begin{array}{l}++ \\
\text { (1) }\end{array}$ & $\begin{array}{l}0 /+ \\
(3)\end{array}$ & $\begin{array}{l}-\cdot \\
\text { (2) }\end{array}$ & $\begin{array}{l}\text { Stratégie } \\
\text { risquée }\end{array}$ \\
\hline
\end{tabular}

Compatibilité des options stratégiques avec les scénarios d'environnement : de ++ (adéquat) à - (incompatible) ; entre parenthèses : rang de l'option pour chaque scénario.

Quelles leçons tirer de cet exercice ? Le travail de réflexion prospectif a permis de disposer de la représentation de futurs possibles (options stratégiques) face à un environnement complexe et incertain (scénarios). II montre la nécessité de développer une aptitude au changement et de bâtir une stratégie flexible permettant de faire face avec succès aux évolutions du contexte. Lors de la création du groupe prospective et stratégie, le directeur général avait décidé qu'il serait composé de chercheurs en position d'encadrement intermédiaire et qu'aucun membre de la direction ne participerait aux travaux afin de laisser toute liberté de parole et de favoriser la créativité. La composition du groupe prospective et stratégie était donc différente de celle du groupe de cadres dirigeants et de chercheurs qui a lancé le projet d'entreprise. Ce choix a permis effectivement de motiver des chercheurs, d'insuffler un réflexe prospectif dans certains programmes et d'établir des réseaux à l'intérieur de l'organisme ainsi qu'avec des chercheurs d'autres institutions.

Mais la méthode choisie a eu des limites. Tout d'abord, le développement de compétences en prospective et en stratégie est long. Cet investissement doit être fait sur de nombreux individus. Certains ont une capacité d'anticipation et d'analyse plus ou moins naturelle. Pour la plupart, il est nécessaire d'être impliqué dans de nombreuses études et de travailler avec d'autres chercheurs (d'où l'importance des réseaux) pour développer une vision globale. La deuxième limite réside dans la 
composition du groupe. Le directeur général et plusieurs de ses proches collaborateurs ayant changé pendant la période des travaux, les membres du groupe prospective et stratégie ont été déconnectés des instances de décision. Ils ont fait un travail de prospective créatif, qui a eu du mal à étre approprié par l'ensemble du CIRAD et à être utilisé par la direction générale dans la construction d'un plan stratégique. Cela démontre que la relation entre l'importance accordée par la direction générale à la démarche et l'appropriation par le personnel est très lorte. Aujourd'hui, le CIRAD rencontre des difficultés pour bâtir un plan stratégique, c'est-à-dire élaborer les règles de conduite qui permettraient d'atteindre les objectifs et la grille qui définirait la répartition des ressources. La troisième limite réside dans la déconnection entre les efforts globaux lancés par la direction générale (projet d'entreprise et groupe prospective et stratégie) et les efforts des départements du CIRAD. Ainsi, les départements préparent des schémas pluriannuels de programmation de façon décentralisée et assez bureaucratique. Ces schémas sont souvent davantage une présentation de l'existant que des propositions pour l'avenir en fonction de questions identifiées. Le cadre stratégique qui doit être donné par la direction générale reste imprécis. Les travaux d'anticipation réalisés ne sont pas utilisés.

Préparer demain pas à pas
Les deux exercices (projet d'entreprise et prospective par scénarios) qui ont été menés au CIRAD ont toutefois été fort utiles. Ils ont ouvert l'horizon des chercheurs et leur ont permis de mieux faire face à leurs responsabilités. Ils ont contribué à la notoriété du Centre. La démarche participative d'élaboration du projet d'entreprise a facilité son appropriation par le personnel CIRAD. Les consultants ont apporté un œil extérieur qui permet de mieux se connaître de l'intérieur. Ils ont permis de passer outre les idées reçues et ont donné une méthode de travail. Certes, le CIRAD, ayant encore peu l'habitude de travailler avec des consultants, ne les a peutêtre pas utilisés aussi efficacement qu'ils auraient pu l'être. Leur présence a parfois été davantage ressentie comme permettant de faire face à une absence de compétence interne que comme un investissement. Par ailleurs, étant donné la complexité de l'environnement, les moyens et le temps limités, les méthodes ont été adaptées aux besoins du CIRAD, c'est-à-dire simplifiées et raccourcies. Cependant, les nombreuses consultations externes ont montré que le CIRAD change. Un réseau socio-économique favorable (Callon, 1989) a été constitué et il est essentiel dans le processus d'innovation.

Le projet d'entreprise a donné des réponses à deux questions souvent posées : à quoi voulons-nous servir ? Quelle entreprise voulons-nous être ? La réflexion prospective a permis d'examiner l'environnement macro (économique, social, sociologique, politique, etc.), l'environnement du secteur, les alliés potentiels, et de commencer à déterminer les compétences fondamentales du CIRAD. Ce travail peut aider l'organisme à saisir les opportunités majeures de son environnement et à s'appuyer sur ses atouts, tout en écartant certaines menaces et en compensant ses faiblesses les plus graves. En effet, il est important pour les entreprises de recentrer leurs activités autour de leurs compétences distinctives et de concentrer leurs efforts sur leur mise en œuvre et leur 
public de recherche, le CIRAD

renforcement (Hamel et Prahalad, 1995).

Le projet d'entreprise du CIRAD et le groupe prospective et stratégie ont eu des retombées indirectes sur le plan international. Les réflexions menées par le CIRAD en relation avec des partenaires extérieurs ont contribué à faire avancer les débats dans d'autres instances. En effet, plusieurs membres des deux groupes internes au CIRAD faisaient en même temps partie d'autres groupes de réflexion. Les idées développées par le CIRAD ont ainsi pu circuler largement et être appropriées et améliorées par d'autres. On peut penser que l'évolution récente de la Conférence des responsables de recherche agronomique africains (CORAF), la réflexion européenne sur la recherche agronomique pour le développement, les conclusions du Comité national d'évaluation de la recherche (CNER), les travaux du Comité national de coordination pour la recherche au service du développement (CNC), et même les réflexions menées au sein du Groupe consultatif pour la recherche agricole internationale (GCRAl) ont été en partie inspirés et nourris par les réflexions du CIRAD.

Aujourd'hui, au CIRAD, le défi est le passage de la réflexion à l'action. Les organismes de recherche rencontrent parfois des difficultés à effectuer ce passage pour plusieurs raisons. Premièrement, les directeurs des établissements de recherche ont souvent une faible culture managériale car ils sont généralement issus du sérail. Ce sont davantage des chercheurs que de véritables entrepreneurs. Or, comme le souligne Michel Crozier (1994), la recherche est devenue la clef du développement économique et même social et culturel de toutes nos sociétés. Elle met en jeu désormais des sommes très considérables. II paraît donc indispensable de gérer les investissements qu'elle demande de façon efficace. Deuxièmement, les méthodes de programmation de la recherche tentent souvent de programmer les investissements et les résultats. Or, on peut programmer les premiers, mais rarement les seconds. Michel Crozier propose de se concentrer sur le rôle de l'individu et du groupe, la gestion des interfaces entre différentes activités, l'évaluation des résultats et la gestion des carrières.

La difficulté du passage de l'anticipation à l'action a pour troisième raison le fait qu'il existe une ambiguilté entre recherche et prospective. Pour la plupart des entreprises, la prospective et l'analyse stratégique sont des outils qui permettent d'établir une stratégie. Mais, par ailleurs, quelques organisations ont pour seule mission de faire de la prospective et de réfléchir à l'avenir. Les organismes de recherche considèrent souvent qu'ils ont pour mission de réfléchir à l'avenir et de le préparer. lls ont tendance à réfléchir et à ne pas agir ni choisir leur stratégie. Si les organismes de recherche français réfléchissaient sur leurs compétences distinctes, leurs spécificités, cela les aiderait peut-être à mieux définir leur stratégie et à se mobiliser autour d'elle.

Enfin, la réussite ou l'échec étant difficiles à mesurer (il n'y a pas un indice aussi simple que le chiffre d'affaires), la capacité des responsables de la recherche à admettre la réalité reste faible. Cette situation renforce la probabilité du scénario tendanciel, car ne pas reconnaître la réalité conduit à se laisser dominer par les tendances les plus fortes et à ne pas lutter contre elles pour créer son propre avenir. 
Des actions en cours
En 1996, la direction générale du CIRAD a pris deux décisions importantes, conséquences des processus précédents. En premier lieu, elle a lancé un projet destiné à adapter au CIRAD les principes de la gestion par activités et par processus (Lorino, 1991 ; Zarifian, 1995). Ce projet doit à terme aider à la programmation des activités et au partage des responsabilités ; il doit faciliter le suivi comptable et le contrôle de gestion. II s'agit de reconnaître les activités particulières à la recherchedéveloppement et les autres activités non spécifiques à la mission du CIRAD, et de distinguer les processus créateurs de valeur et les processus d'appui.

En second lieu, un observatoire du changement a été créé au sein de l'organisme, pour animer, appuyer sur le plan méthodologique les activités de prospective et d'analyse stratégique, et pour en diffuser les résultats. Cet observatoire du changement a pour mission d'aider à formuler des recommandations stratégiques pour la direction du CIRAD par des études à caractère prospectif et par des synthèses et d'apporter un appui méthodologique aux unités du CIRAD pour conduire des exercices de prospective et des analyses stratégiques devant déboucher sur une identification des priorités et une programmation des activités. II doit aider la direction générale à alimenter, à partir des travaux conduits, la réflexion des tutelles et des institutions concernées par la recherche agronomique tropicale et le développement des pays du Sud. En fonctionnant uniquement avec un réseau de correspondants au sein du CIRAD, l'observatoire du changement doit diffuser dans la culture des agents du CIRAD un comportement d'anticipation ouvert sur un avenir multiple et incertain permettant de concevoir le futur que l'institution souhaite se donner. 


\section{BIBLIOGRAPHIE}

AUBERT C. (1995), "Hamel et Prahalad, mode d'emploi", L'Expansion Management Review, juin 1995. pp. 102-109.

BENASSOULI P. et MONTI R. (1995), "La planification par scénarios. Le cas d'Axa France 2005", Futuribles, novembre 1995, pp. 37-61.

BRECHET J.P. (1994), "Du projet d'entreprise au projet d'entreprendre", Revue française de gestion, $n^{\circ} .99$, juin-août 1994, pp. 5-14.

CALLON M. (sous la direction de) (1989), La science et ses réseaux : genèse et circulation des faits scientfiques, Paris, Editions La Découverte, 1989, 214 pages.

CIRAD (1990), Un projet d'entreprise pour le CIRAD. Quel avenir ? Quelle stratégie ? mai 1990,58 pages.

CIRAD (1991), Le projet d'entreprise du CIRAD. Renouveler notre coopération dans un monde qui change. CIRAD, 1991, 108 pages.

CIRAD (1991), Les journées du projet d'entreprise. CIRAD, Montpellier, 12-13 septembre 1991, 84 pages.

CIRAD (1995), Prospective et options stratégiques pour le CIRAD. Rapport du groupe prospective et stratégie. CIRAD, Notes et Documents $n^{\circ}$ 19, 1995, 124 pages.

COMITE NATIONAL D'EVALUATION DE LA RECHERCHE (CNER). Evaluation du Centre de coopération internationale en recherche agronomique pour le développement (CIRAD). Rapport d'instruction, novembre 1995, 183 pages.

CROZIER M. (1994), "Peut-on manager la recherche et linnovation ?", Politiques et management public, volume 12, $n^{\circ} 2$, juin 1994. pp. 11-16.

CROZIER M. et FRIEDBERG E. (1977), L'acteur et le système : les contraintes de l'action collective, Paris, Editions du Seuil, Collection Points, 1977, 436 pages.

GIGET M. (1989), "L'identité de l'entreprise. Préalable à la réflexion stratégique", Futuribles, novembre 1989, pp. 15-29.

GODET M. (1991), De l'anticipation à l'action, Paris, Dunod, 1991, 390 pages. 
GODET M. et alii (1991), "Problèmes et méthodes de prospective : boite à outils", Futuribles-GERPA, deuxième édition, 1991, 73 pages.

GONDRAND F. (1987), "Un projet d'entreprise : pour quoi faire ?", Problèmes économiques, $\mathrm{n}^{\circ} 2066,16$ mars 1988, pp. 24-30.

HAMEL G. et PRAHALAD C.K. (1995), La conquéte du futur. Stratégies audacieuses pour prendre en main le devenir de votre secteur et créer les marchés de demain, Paris, InterEditions, 1995, 325 pages.

HERNANDEZ .M. (1994), "Le projet d'entreprise - effet de mode ou instrument de changement ?", Problèmes économiques, n² 2 401, 7 décembre 1994, pp. 13-19.

de JOUVENEL H. (1993), "Sur la démarche prospective, un bref guide méthodologique", Futuribles, septembre 1993, pp. 51-70.

de LATTRE M. (1990), La gestion stratégique des relations des instituts de recherche avec leur environnement. Le cas des instifuts de recherche agricole du Cameroun et du Sénégal. Thèse de doctorat. Université de Paris X, octobre 1990, 310 pages.

LESOURNE J. (1989), "Plaidoyer pour une recherche en prospective", Futuribles, novembre 1989, pp. 85-89.

LESOURNE J. et STOFFAES C. (1996), La prospective stratégique d'entreprise. Concepts et études de cas, Paris, InterEditions, 1996, 276 pages.

LORINO P. (1991), Le contróle de gestion stratégique. La gestion par activités, Paris, Dunod, 1991.

MARTINET A.C. (1984), Management stratégique, organisation et politique, McGraw Hill, 1984, 126 pages.

MASSE P. (1965), Le plan ou l'anti-hasard, Paris, Gallimard, 1965, 250 p.

PONSSARD J.P. (1993), "Gérer la recherche-développement comme un défi : quel rôle pour la planification ?", Cahiers d'économie et sociologie rurale, $n^{\circ} 28,1993$.

THIETART R.A. (1984), Stratégie d'entreprise, McGraw Hill, 1990, 194 pages.

REITTER R. et RAMANANTSOA B. (1985), Pouvoir et politique : au-delà de la culture d'entreprise, McGraw Hill, 1985, 104 pages.

SCHWARTZ P. (1993), "La planification stratégique par scénarios", Futuribles, mai 1993, pp. 31-50. 
STRATEGOR (1988), Stratégle, structure, décision, Identité, Paris, InterEditions, 1988, 511 pages.

ZARIFIAN P. , "La gestion par activités et par processus à la croisée des chemins", Gérer et comprendre, mars 1995. 